\title{
Effective Pixel Classification of Mars Images Based on Ant Colony Optimization Feature Selection and Extreme Learning Machine
}

\author{
Abdolreza Rashno ${ }^{1 *}$, Behzad Nazari ${ }^{2}$, Saeed Sadri $^{3}$ and Mohamad Saraee ${ }^{4}$ \\ 1*. Department of Electrical and Computer Engineering, Isfahan University of Technology, Isfahan, 84156-83111, Iran (e-mail: \\ a.rashno@ec.iut.ac.ir), Corresponding author. \\ 2. Department of Electrical and Computer Engineering, Isfahan University of Technology, Isfahan, 84156-83111, Iran (e-mail: \\ nazari@cc.iut.ac.ir) \\ 3. Department of Electrical and Computer Engineering, Isfahan University of Technology, Isfahan, 84156-83111, Iran (e-mail: sadri \\ @cc.iut.ac.ir). \\ 4. School of Computing, Science and Engineering, University of Salford-Manchester, United Kingdom (e-mail: \\ m.saraee@salford.ac.uk).
}

\begin{abstract}
Mars rover, a robot which explores the Mars surface, is the process of automatic segmentation of images taken by front-line anoramic camera (Pancam). This procedure is highly significant since the transformation cost of images from Mars to earth is extremely high. Also, image analysis may help Mars rover for its navigation and localization. In this paper, a new feature vector including wavelet and color features is presented for Mars images. Then, this feature vector is presented for extreme learning machine (ELM) classifier which leads to a high accurate pixel classifier. It is shown that this system statistically outperforms support vector machine (SVM) and k-nearest neighbours (KNNs) classifiers with respect to both accuracy and run time. After that, dimension reduction in feature space is done by two proposed feature section algorithms based on ant colony optimization (ACO) to decrease the time complexity which is very important in Mars on-board applications. In the first proposed feature selection algorithm, the same feature subset is selected among the feature vector for all pixel classes, while in the second proposed algorithm, the most significant features are selected for each pixel class, separately. Proposed pixel classifier outperforms prior methods by $6.44 \%$ and $5.84 \%$ with respect to average Fmeasure and accuracy, respectively. Finally, proposed feature selection methods decrease the feature vector size up to $76 \%$ and achieves Fmeasure and accuracy of $91.72 \%$ and $91.05 \%$, respectively, which outperforms a conventional method with $87.22 \%$ and $86.64 \%$.
\end{abstract}

Keywords: pixel classification; feature selection; ant colony optimization; extreme learning machine; wavelet features; color features.

\section{INTRODUCTION}

Exploration and research in Mars has been increased significantly over the last decade. Geological and structural information of Mars conducted by robots are all the results of such explorations. Also, mineral classification of mars images presents valuable information of Mars environment [1]. Furthermore, the extraction of chemical and mineralogical properties of the Mars stones and soil could be helpful in the future researches and explorations [2].

Recently the automated robot, referred as Mars rover is equipped with a front-line panoramic camera (Pancam) instrument. A great deal of information can be extracted and organized from Mars images. The transformation cost from Mars to earth is very high in the term of Mars rover supply power. Therefore, automated image analysis systems with a high degree of accuracy are required [3]. The main task in these systems is the classification of rocks and other objects in the images. This procedure is so important because it avoids sending the irrelevant and redundant images to the earth. It also helps Mars rover for navigation and localization in the Mars surface. The rocks in Mars have diverse colors and textures, covered by dust soil with various intensities, rotation and scale. In addition, these images are blurred by instrument movement and are contaminated by environment noise. In [4], a theorem for the efficient segmentation and detection of motion blur was presented which led to the classification of pixels as either blurred or unblurred. Furthermore, in [5], a new kernel-specific feature vector with a good discrimination property was proposed and then presented for the classifiers to detect different types of blur including motion and defocus blurs. All of the above items address the main challenges in Mars images analysis [6].

Our goal in this research is to develop a new method for automatic pixel classification in Mars images. The first step in this method is extraction of a set of features from image pixels. There are many features proposed in the literature for accurate classification of the pixels [7 and 8]. Increasing dimension of feature set leads to more computational time which is not acceptable in our interest, especially in onboard applications such as Mars rover, with its limited amount of power, memory and processing rate. On the other hand, there is an opinion that not all extracted features are relevant in classification and these extracted features decrease the accuracy [9]. A good feature vector should be easily extractable from the images, discriminative, robust against noise and distortion. A key issue is that the feature vector must be short and efficient. Most of the classification methods presented for Mars rover involve high computational costs because of high dimensionality of feature vectors [3]. Some feature selection approaches are ant colony optimization (ACO) [10], particle swarm optimization (PSO) [11], genetic algorithm (GA) [12], feature selection with harmony search [13] and so on. Many feature selection approaches have been proposed for different applications such as face recognition [14], data mining and pattern recognition [15], speaker verification [16] and classifier ensemble reduction [17]. Feature selection has been applied in Mars image classification in the term of minimizing feature subset by fuzzy-rough feature selection [3 and 21] and information gain ranking technique [18]. 
The main contributions of this paper that lead to a high accurate pixel classifier for the classification of different types of rocks and sands from their surrounding background in Mars images are summarized as follows:

1) The texture and structure of difference types of rocks in Mars images are analyzed and then a new feature set for pixel description is proposed. The feature set includes texture and color features and makes difference between all types of rocks and lead to better pixel classification. For texture features, statistical parameters of LH and HL components in wavelet domain are considered. This idea is very useful for distinguishing rocks with layered and waved structures since wavelet decomposition provides vertical and horizontal frequency components of images. Dominant color descriptor, local color histogram and color statistic features are all used as color features. These features are useful for describing structures such as small black stone and sand, dark large size rocks with shadow and flat rocks. Then, a recent efficient classifier referred as extreme learning machine (ELM) is used for pixel classification. We will show that this classifier with the proposed feature set not only lead to a high accurate pixel classifier but also outperforms other classifiers such as SVM and KNNs with respect to both accuracy and run time.

2) Because of the limitation of the power, memory and processing rate in on-board applications such as Mars rover for Mars image analysis, dimension reduction with preserving classification accuracy is addressed as another contribution of this research. For this goal, two feature selection methods based on ACO are proposed to select the most relevant and significant features from the complete feature set. For feature selection methods a new idea named as feature grouping is proposed and it's efficiency is shown for feature selection algorithms. Applying these feature selection methods in pixel classification, decreases the computational time and preserves classification performance simultaneously not only in our proposed system with ELM but also in SVM and KNNs classifiers. The first feature selection method selects the same feature subset for all pixel classes while the second one presents a feature subset for each pixel class separately. Both feature selection methods, with the same degree, decrease the run time of classifiers in both train and test phases considerably. The first proposed method preserves the classification accuracy with just a little decrease while the second one increases it.

3) The pixel classification accuracies of ELM, SVM and KNNs classifiers with different parameters and different feature subsets (complete feature set, feature subsets found by the first and second proposed methods and genetic algorithm) will be shown quantitatively followed by statistical interpretations of the results.

The rest of this paper is organized as follows: Section 2 describes the extraction of feature set from image pixels. Pixel classifiers are explained in more details in Section 3 and 4. Our two proposed feature selection methods are described completely in Section 5. Experimental setup and results are described in Sections 6 and 7 respectively. Finally, the conclusion and future works are discussed in Section 8.

\section{FEATURE EXTRACTION}

Converting complex image data to their corresponding feature vector is the task of feature extraction. Feature vectors represent a large set of data accurately as well as simplifying the amount of memory and computation cost required for data processing [19]. The main purpose of this research is pixel classification. Corresponding feature vector for each pixel is extracted from a surrounding window of that pixel. Proposing features with better pixel representation is a challenging task. For this task, many experiments were performed and the following features are proposed. Some types of layered rocks contain horizontal and vertical frequency components. A very good descriptor for these types of rocks is the texture features extracted from wavelet transform. Therefore, statistical parameters in wavelet domain are used as pixel representation. Other rock types such as rover tracks (which have a darker surface in comparison with other rocks), Small black stone and sand flat rocks could be easily identified by color features. Finally, a feature set including wavelet coefficient features, dominant color descriptor features, local color histogram features and color statistic features is proposed to be used in this experiment and is explained in the next sections.

\subsection{Wavelet Features}

Each pixel of the input image is windowed and a one level two-dimensional wavelet decomposition of the window is obtained. Then Euclidean norm is incorporated to the wavelet coefficients of rows and columns of LH and HL, separately. Finally, the mean and variance of Euclidean norms of rows and columns are calculated which leads to 8 parameters.

\subsection{Color Features}

Color features are considered as low-level features which are very stable and robust in comparison with other image features such as texture and shape. Since, these features are not sensitive to rotation, translation and scale changes; they could be so applicable to pixel classification of Mars images which are noisy and degraded by rotation and camera transformations. In this work, we have used the dominant color descriptor (DCD) features, local color histogram features and color statistic features.

\subsubsection{Dominant Color Descriptor}

DCD is one of the approved color descriptors in the MPEG-7 Final Committee Draft among several number of histogram descriptors [20]. Both representative colors and the percentage of each color are included in DCD. In DCD, colors of an image are divided into a number of partitions named coarse partitions. All points in a partition are assumed to be similar. Partition centers are the average value of all pixel colors in each partition and are calculated with by Eq. 1.

$$
C_{j}=\frac{\sum_{p \in P_{i}} p}{\sum_{p \in P_{i}} 1}
$$


In which $P_{i}$ is the ith partition. In this research, the DCD features are extracted in RGB domain. Each pixel color is assigned to a partition and its color is replaced by the center value of that partition. Therefore, the image colors are quantized. For each pixel, the DCDs of a window around that pixel are calculated and presented as a feature vector corresponding to it. We assumed 8 color dominants in this research. In Fig. 1, the right image is an image in RGB domain and the left image shows the result of applying DCD.
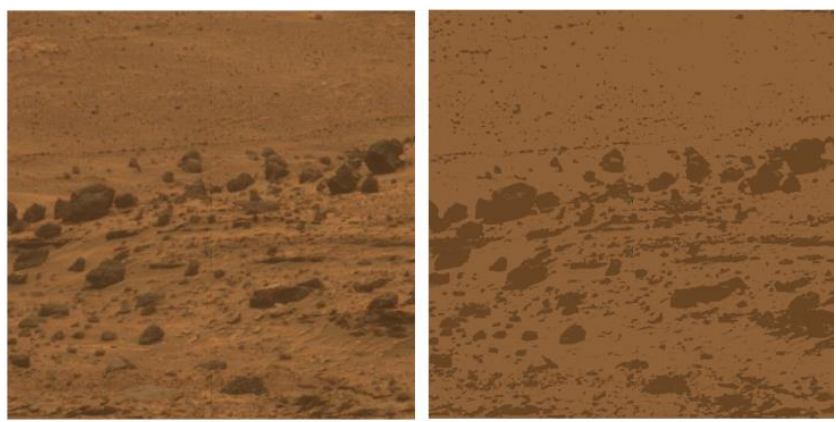

Fig. 1. DCD of an image in RGB color space.

\subsubsection{Local Color Histogram Features}

Histogram is the discrete statistical probability density of the image [21]. Mars images are in RGB domain with the range 0-255 in each component. If all 256 bins are considered per R, G and B components in each histogram, it will be computationally expensive. Accordingly the range is divided to 8 equal sub-ranges for each component. To localize these features, a window is considered around each pixel and histogram of the window is computed. Therefore, each pixel of the image is mapped to a 24-dimensional feature vector in the term of local color histogram features.

\subsubsection{Color Statistic Features}

The first and the second moments of a window around pixels are mean and standard deviation, respectively. These features are also applied in RGB domain. The mean and standard deviation corresponding to each pixel are considered as color statistic features for each component (R, G and B). Table.1 shows the abbreviation names of all extracted features that will be used in this paper.

TABLE 1

AbBreviation Names of All Extracted Features.

\begin{tabular}{|l|l|l|l|l|l|}
\hline \multicolumn{1}{|c|}{ Wavelet } & \multicolumn{1}{c|}{ Feature Meaning } & \multicolumn{1}{c|}{ Color } & Feature Meaning & \multicolumn{1}{c|}{ Color } & Feature Meaning \\
\hline LH_meanL & Mean of norms of L Components of LH & DCDR $_{\mathbf{1 - 8}}$ & Dominant colors of R & $\boldsymbol{B}_{s}$ & Std of B component \\
\hline LH_meanH & Mean of norms of H Components of LH & DCDG $_{\mathbf{1 - 8}}$ & Dominant colors of G & $\boldsymbol{R}_{\mathbf{1}-\mathbf{8}}$ & Histogram bins of R component \\
\hline LH_stdL & Std of norms of L Components of LH & DCDB $_{\mathbf{1 - 8}}$ & Dominant colors of B & $\boldsymbol{G}_{\mathbf{1}-\mathbf{8}}$ & Histogram bins of G component \\
\hline LH_stdH & Std of norms of H Components of LH & $\boldsymbol{R}_{\boldsymbol{m}}$ & Mean of R component & $\boldsymbol{B}_{\mathbf{1}-\mathbf{8}}$ & Histogram bins of B component \\
\hline HL_meanH & Mean of norms of L Components of HL & $\boldsymbol{G}_{\boldsymbol{m}}$ & Mean of G component & & \\
\hline HL_meanL & Mean of norms of L Components of HL & $\boldsymbol{B}_{\boldsymbol{m}}$ & Mean of B component & & \\
\hline HL_stdH & Std of norms of H Components of HL & $\boldsymbol{R}_{\boldsymbol{s}}$ & Std of R component & & \\
\hline HL_stdL & Std of norms of L Components of HL & $\boldsymbol{G}_{\boldsymbol{s}}$ & Std of G component & & \\
\hline
\end{tabular}

3. PIXEL Classifier By SVM

There are different pixel classes in Mars images. Extracted features should be presented for classifiers to train model parameters in classification. For each pixel class, a one-against-all binary SVM is trained. Pixels of the main class are assumed as positive and pixels of all other classes are assumed as negative samples. The main problem is that the number of pixels in the main class is much less than the number of pixels of the other classes. This leads our model to unbalanced train data in SVM classifier and the model may be biased to negative samples. We address this issue by random selection of $k$ samples among all negative samples and then introducing selected samples as training data of negative class. Fig. 2 shows the result of the process of train data balancing for SVM classification. 


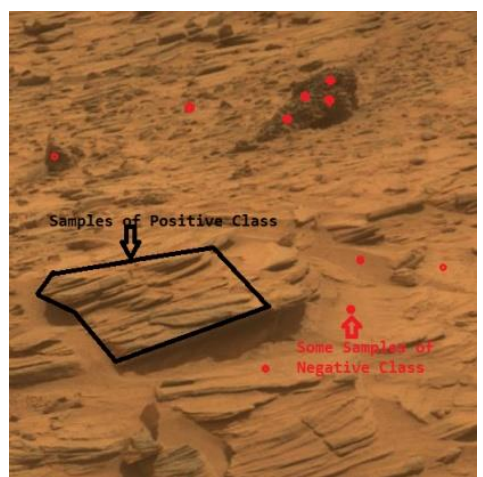

Fig. 2. Balancing train data for SVM classification.

\section{4.}

\section{PIXEL Classifier By ELM}

ELM originally stems from biological learning and is first proposed for the single-hidden layer feed-forward neural networks (SLFNs) and then for the generalized single-hidden layer feed-forward networks [22]. The output function of the ELM for generalized SLFNs case is computed by Eq. 2 .

$f_{L}(x)=\sum_{i=1}^{L} \beta_{i} h_{i}(x)$

where $\beta_{i}$ is a weight between $i$ th hidden node and the output nodes and $h_{i}(x)$ is the output of the $i$ th hidden node. It is clear that the output functions of the hidden nodes could be different. In general, $h_{i}(x)$ is a non-linear piecewise continuous function such as sigmoid, hardlimit, gaussian and multi-quadratic.

The feed-forward neural networks try to reach to smaller training errors. In ELM, both the smaller training error and the smaller norm of output weights are considered. The smaller norm of weights, the better generalization performance could be achieved. So, ELM has a higher performance in comparison with classic feed-forward networks. ELM could be expressed as an optimization problem in Eq. 3.

$\min :\|\beta\|_{p}^{\sigma_{1}}+C\|H \beta-T\|_{q}^{\sigma_{2}}$

where $\sigma_{1}$ and $\sigma_{2}$ are positive values and $p, q=0,0.5,1,2, \ldots . \mathrm{H}$ is the matrix of $h$ functions named as hidden layer output matrix and $\mathrm{T}$ is the training data target matrix. The learning rules and mathematical theory of ELM are discussed in deeper details in [23 and 24].

ELM can be used in both classification and regression. Here, similar with SVM, ELM is used for pixel classification with a one-againstall classification scheme. For this task, ELM is trained with the training set of pixel feature vectors and then the feature vectors of unseen pixels are presented for classification. The similar idea for train data balancing, which was proposed for SVM, is applied here, too. We have used the ELM toolbox in MATLAB for ELM classification ${ }^{1}$.

\section{FEATURE SELECTION}

Feature selection is a discrete optimization problem which selects $m$ features among $n$ ones [26]. The whole search space includes all possible feature subsets of the main feature set. The number of all possible subsets is computed by Eq. (4).

$$
\sum_{s=0}^{n}\left(\begin{array}{l}
n \\
s
\end{array}\right)=\left(\begin{array}{l}
n \\
0
\end{array}\right)+\left(\begin{array}{l}
n \\
1
\end{array}\right)+\cdots+\left(\begin{array}{l}
n \\
n
\end{array}\right)=2^{n}
$$

where $n$ represents feature vector dimension and $s$ is the size of the current feature subset.

Alleviating the effect of the dimensionality, enhancing generalization capability, improving model interpretability and speeding up the learning process are all the benefits of removing irrelevant and redundant features from the main features [26 and 27]. Two types of feature selection methods are filter and wrapper methods. Filter methods use some metrics such as inter-class distance, probabilistic distance, class separability, entropy, error probability, consistency and correlation. In wrapper methods, classification accuracy is used for feature evaluation, show that the classifier model should be trained and tested in each step. In the filter methods, feature subset evaluation does not depend on the classification model because it does not need to train the system for each feature subset. On the other hand, wrapper methods are computationally expensive and have a risk of model over-fitting. As a result, filter methods have better generalization as well as lower complexity and over-fitting, although generally wrapper methods usually have a higher accuracy [28, 29 and 30].

\footnotetext{
${ }^{1}$ http://www.ntu.edu.sg/home/egbhuang/elm_codes.html
} 


\subsection{Genetic Algorithm for Feature Selection}

GA introduced by Holland in 1975, is a randomized heuristic search technique based on biological evolution strategies. Many complex optimization problems could be solved by GA in which chromosomes represents candidate solutions in a large population. Initial chromosomes (solutions) usually are created randomly and then next generations are generated from chromosomes with higher fitnesses. GA has been applied to our problem of interest as feature selection and feature weighting. A binary vector with the same size of the feature vector is a chromosome (solution). If the corresponding bit of a feature is one, that feature is selected and it is dropped in case it is zero. The purpose of GA in feature selection task is to find an optimal binary vector with the smallest number of $1 \mathrm{~s}$ such that the performance of the classifier is maximized [19 and 30]. Another application of GA is feature weighting which assigns numerical weights to features instead of binary weights zero and one [31].

\subsection{Ant Colony Optimization for Feature Selection}

Ant colony optimization (ACO) is an iterative and probabilistic meta-heuristic method. It simulates the natural behavior of ants, consisting of mechanisms of adaptation and cooperation [32]. The ants travel among nodes of a graph and deposit pheromone for finding the shortest path. As pheromone decays over the time, each ant probabilistically prefers to follow a direction with the higher pheromone density. Accordingly the shortest path on the graph will has more pheromone and higher chance to be selected by ants and will be reinforced while pheromone of other paths' diminished [33]. Similar to GA, ACO can be reformulated for feature selection problem by finding a path with minimum cost on a graph. In contrast with classic ACO, here, nodes in the graph represent features and the edges between nodes denote the choice of the next feature [34]. ACO starts to search for the optimal feature subset with the ant's traverse through the graph until a minimum number of nodes are visited and traversal stop criterion is satisfied i.e. the minimum number of features with the highest classification accuracy are achieved. Any feature is allowed to be selected in each step of ant traverse, so the graph is fully connected. The pheromone update rule and transition rule of ACO algorithm can be used by a little reformulation for feature selection. In this case, pheromone and heuristic values are associated to nodes (features) rather than edges. The probability that ant $k$ selects feature $i$ at time step $t$ is computed from Eq. (5).

$$
P_{i}^{k}(t)=\left\{\begin{array}{c}
\frac{\left|\tau_{i}(t)\right|^{\gamma}\left|\eta_{i}\right|^{\delta}}{\sum_{u \in J^{k}}\left|\tau_{u}(t)\right|^{\gamma}\left|\eta_{u}\right|^{\delta}} \quad \text { if } i \in J^{k} \\
0 \quad \text { otherwise }
\end{array}\right.
$$

where $J^{k}$ is the set of features that are allowed to be added to the partial solution in case that they haven't been visited so far. $\tau_{\mathrm{i}}(\mathrm{t})$ and $\eta_{\mathrm{i}}$ are the pheromone value and heuristic desirability associated with feature $i$ respectively. $\gamma$ and $\delta$ are weights of pheromone value and the heuristic information respectively[35]. The amount of deposited pheromone of ant $k$ on feature $i$ in step $t$ is obtained by Eq. (6).

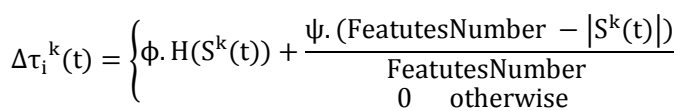

where FeatutesNumber is the number of all features, $S^{\mathrm{k}}(\mathrm{t})$ and $\left|\mathrm{S}^{\mathrm{k}}(\mathrm{t})\right|$ are the feature subset and its length found by ant $k$ at iteration $t$, respectively. $H\left(S^{k}(t)\right)$ is the evaluation of subset $S^{k}(t)$ which is the classifier performance in this literature, $\phi$ and $\psi$ are parameters that determine the importance of the classifier performance and feature subset length, respectively. Finally, the node pheromone is updated by Eq. (7).

$\tau_{i}(t+1)=(1-\rho) \cdot \tau_{i}(t)+\sum_{k=1}^{m} \Delta \tau_{i}^{k}(t)+\Delta \tau_{i}{ }^{g}(t)$

where $m$ is the number of ants, $\rho$ is an evaporation rate which is constant and $g$ is the best ant in previous iteration. It means that nodes pheromone are affected by all ants and more affected by the best ant which deposits additional pheromone on nodes. This causes the search of ants to stay around the optimal solution in the next iterations [27]. ACO feature selection is shown in Fig. 3.

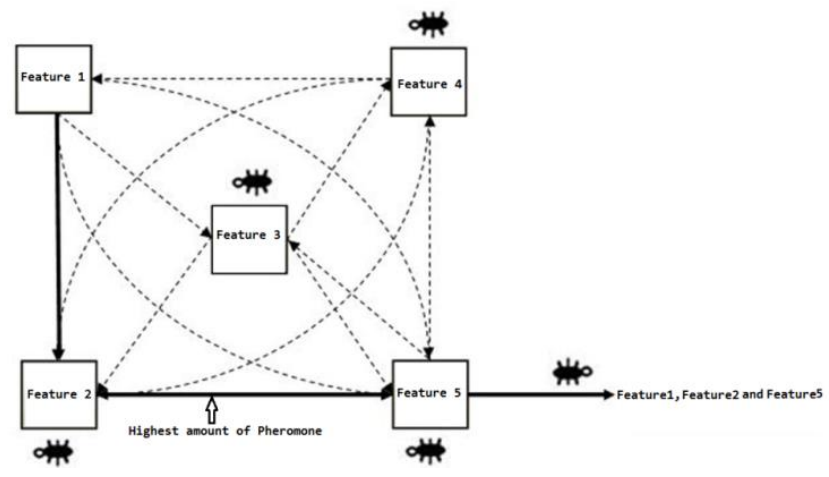

Fig. 3. ACO feature selection. 


\subsection{The Proposed Feature Selection Methods based on ACO}

Selecting the most relevant features and dropping irrelevant ones not only decreases feature vector dimension and computation cost of classifiers but also increases accuracy of such systems. In the proposed pixel classification system, feature selection is done after feature extraction stage. In feature extraction stage, a feature vector is extracted corresponding to each pixel, from a window around that pixel. In this research, two feature selection methods are proposed based on ACO.

\subsubsection{The first method}

It is clear that ACO feature selection is a wrapper method. In conventional ACO feature selection, each ant selects features in its path based on thier efficiency. Suppose that there are $n$ features in feature vector. Each ant is assigned an initial feature randomly and then train the model $n$ - 1 times to select the second feature. After that, $n-2$ times of model training are required for the selection of the third feature. This continues until the ant exit from its traverse. In the worst case, each ant trains the model $\frac{\mathrm{n}(\mathrm{n}-1)}{2}$ times in each iteration. The computation cost is explosively high. Although the feature selection is done only once in the offline phase, we propose a new procedure to decrease the feature reduction cost while preserving the accuracy of classification.

At each iteration, there is a high computational cost to evaluate all 'non-visited' features to select one of them. Experiments show that there is no need for each ant to do that. To remedy this issue we divided features into 13 groups as shown in Table 2.

TABLE 2

GROUPING FEATURES OF A PIXEL IN ACO FEATURE SELECTION.
\begin{tabular}{|l|l|l|l|}
\hline Group Name & \multicolumn{1}{|c|}{ Group Features } & Group Name & \multicolumn{1}{c|}{ Group Features } \\
\hline Group 1 & LH_meanL and LH_stdL & Group 8 & $\boldsymbol{R}_{\boldsymbol{m}}$ and $\boldsymbol{R}_{\boldsymbol{s}}$ \\
\hline Group 2 & LH_meanH and LH_stdH & Group 9 & $\boldsymbol{G}_{\boldsymbol{m}}$ and $\boldsymbol{G}_{\boldsymbol{s}}$ \\
\hline Group 3 & HL_meanH and HL_stdH & Group 10 & $\boldsymbol{B}_{\boldsymbol{m}}$ and $\boldsymbol{B}_{\boldsymbol{s}}$ \\
\hline Group 4 & HL_meanL nad HL_stdL & Group 11 & $\boldsymbol{R}_{\boldsymbol{i}}:$ All histogram bins of R component \\
\hline Group 5 & DCDR $_{\boldsymbol{i}}:$ All color dominants of R component & Group 12 & $\boldsymbol{G}_{\boldsymbol{i}}:$ All histogram bins of G component \\
\hline Group 6 & DCDR $_{\boldsymbol{i}}:$ All color dominants of G component & Group 13 & $\boldsymbol{B}_{\boldsymbol{i}}:$ All histogram bins of B component \\
\hline Group 7 & DCDR $_{\boldsymbol{i}}:$ All color dominants of B component & & \\
\hline
\end{tabular}

The idea of feature grouping has this benefit that each ant only evaluates just one feature among each group. This is important since selection of each feature involves model training and testing. This idea stems from the fact that features in each group have similar effects in classification accuracy. Therefore, evaluation of all features in each group simultaneously is not necessary. On the other hand, all features of a group have a chance to be selected by ants in the next iterations. For example, if LH_meanL is a selected feature from group 1 at step $k$ by an ant, feature LH_stdL will be a candidate to be selected by that ant from step $k+1$ onwards. This shows that all features have a chance to be selected by ants.

In the first proposed method, feature selection is applied to all pixel classes simultaneously. This means that the feature selection scheme tries to select the most relevant features by maximizing the classification accuracy of all pixel classes simultaneously. Therefore, the same feature subset is selected for all classes.

The following pseudo-code describes the proposed feature selection method in more details:

1. Initialization:

- Set the population size of ants.

- Assign each feature to a node of graph and set a random intensity of pheromone to it.

- Set the maximum number of iterations.

2. Divide the features into groups.

3. Each ant builds its own solution. So, each ant should start its job from a feature(node), this feature is assigned to each ant randomly in this step and marked as 'visited'.

4. For each ant, select one feature from each feature group randomly and save them in array $a$. Among features in $a$, select the feature with the highest probability calculated by Eq. (5) and mark this feature as 'visited'.

5. If the ant has not reached to its proposed threshold in Eq. (8), go to 4.

$$
\text { Ant_Threshold }=\varphi * \exp ^{-\frac{\mathrm{FN}}{\mathrm{N}}}+\omega * \exp ^{\mathrm{F}-\text { Measure }}
$$

where $F N$ is the feature cardinality of the selected feature by the ant so far, $N$ is the number of all features, $\varphi$ and $\omega$ are the parameters that control the effect of feature size and F_measure, respectively with restriction $\varphi+\omega=1$. Afterwards, all ants complete their search as a term of finding feature subsets.

The pheromone update is then started by three rules: 1) each ant deposits some pheromone on features. This idea stems from the natural behavior of ants. 2) Some pheromone is evaporated by time. 3) The best ant has more effect in path pheromone update since it could be a sign for other ants to follow its path in the next steps. The amount of pheromones in all features is affected by the classification accuracy and the number of features which are selected by ants.

6. For all ants, do steps 3-5.

7. Each ant deposits a quantity of pheromone on features which is marked as 'visited' in its path by Eq. (9). 


$$
\Delta \text { pheromone }(\mathrm{i}, \mathrm{k})=\propto .(\operatorname{Model} \operatorname{Accurarcy}(\mathrm{k}))+\beta .\left(\frac{\text { FeaturesNumber-FeaturesNumber }(\mathrm{k})}{\text { FeaturesNumber }}\right)
$$

where $\Delta$ pheromone $(\mathrm{i}, \mathrm{k})$ is the amount of pheromone which is deposited by ant $\mathrm{k}$ on feature $\mathrm{i}$, model accuracy is either Fmeasure or accuracy of the classification model (which will be explained in experimental section) trained by features founded by ant $k$. FeatutesNumber and FeaturesNumber(k) are the number of all features and the number of features in the path of ant $k$ respectively. Finally, $\alpha$ and $\beta$ are two parameters that control the relative weights of classifier performance and feature subset length, respectively, according to $\alpha+\beta=1$.

8. Find the best ant with the highest F-measure.

9. Pheromone update (the pheromone of all features is evaporated, all ants deposit a pheromone on the features in their paths and the best ant has extra effect on pheromone update):

pheromone $_{i}(\mathrm{t}+1)=(1-\rho) \cdot$ pheromone $_{\mathrm{i}}(\mathrm{t})+\sum_{\mathrm{k}=1}^{\mathrm{m}} \Delta$ pheromone $(\mathrm{i}, \mathrm{k})+\Delta$ pheromone$(\mathrm{i}$, BestAnt $)$

10. Previous ants are removed and new ants are generated randomly.

11. If the stop criterion (ants follow the same path or maximum number of iteration is reached) is not achieved (go to 3 , else go to 12

12. The path of ant with maximum F-measure is the best solution.

13. End

The block diagram of the first proposed feature selection algorithm is shown in Fig. 4.

\subsubsection{The second method}

Our first proposed feature selection method returns the most relevant features as optimal feature subset for all seven pixel classes. The first method reduces the feature vector length with maximizing Fmeasure or accuracy (to be defined later). In feature reduction phase of the first method, the main properties of all pixel classes were investigated and a feature vector including wavelet and color features was proposed. This was based on the fact that all pixels in different classes could be classified from other classes by using a single feature vector. It is clear that using an optimal feature vector for each of seven classifiers can improve the performance of the overall classification. For example, horizontal and vertical frequency components of wavelet decomposition in wavelet features are irrelevant feature for classifying pixels of class C7 while these features are strongly relevant features for classifying pixels in class C4 in Fig.5.
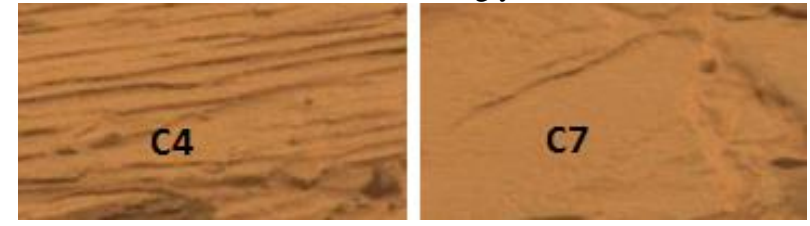

Fig 5. Structure of classes C4 and C7.

Accordingly in the second proposed feature selection method, an optimal feature subset is extracted for each class separately. This idea cannot be applied to ordinary classification methods such as KNNs while it can be applied to one-against-all ELMs and SVMs of all seven classes. Although this is a time consuming task, it is offline which needs to be done only once. The block diagram of this method is depicted in Fig. 6.

This type of feature selection presents an optimal feature subset for each pixel class which increases the classification accuracy in comparison with the complete feature set since all irrelevant and redundant features are omitted from the feature set in each class. The pseudo-code for the second proposed algorithm is presented as follows:

1- For each pixel class do 2-4.

2- Apply the first proposed feature selection.

3- Compute an optimal feature set for each class separately.

4- Train each binary pixel classifier by its own feature set. 


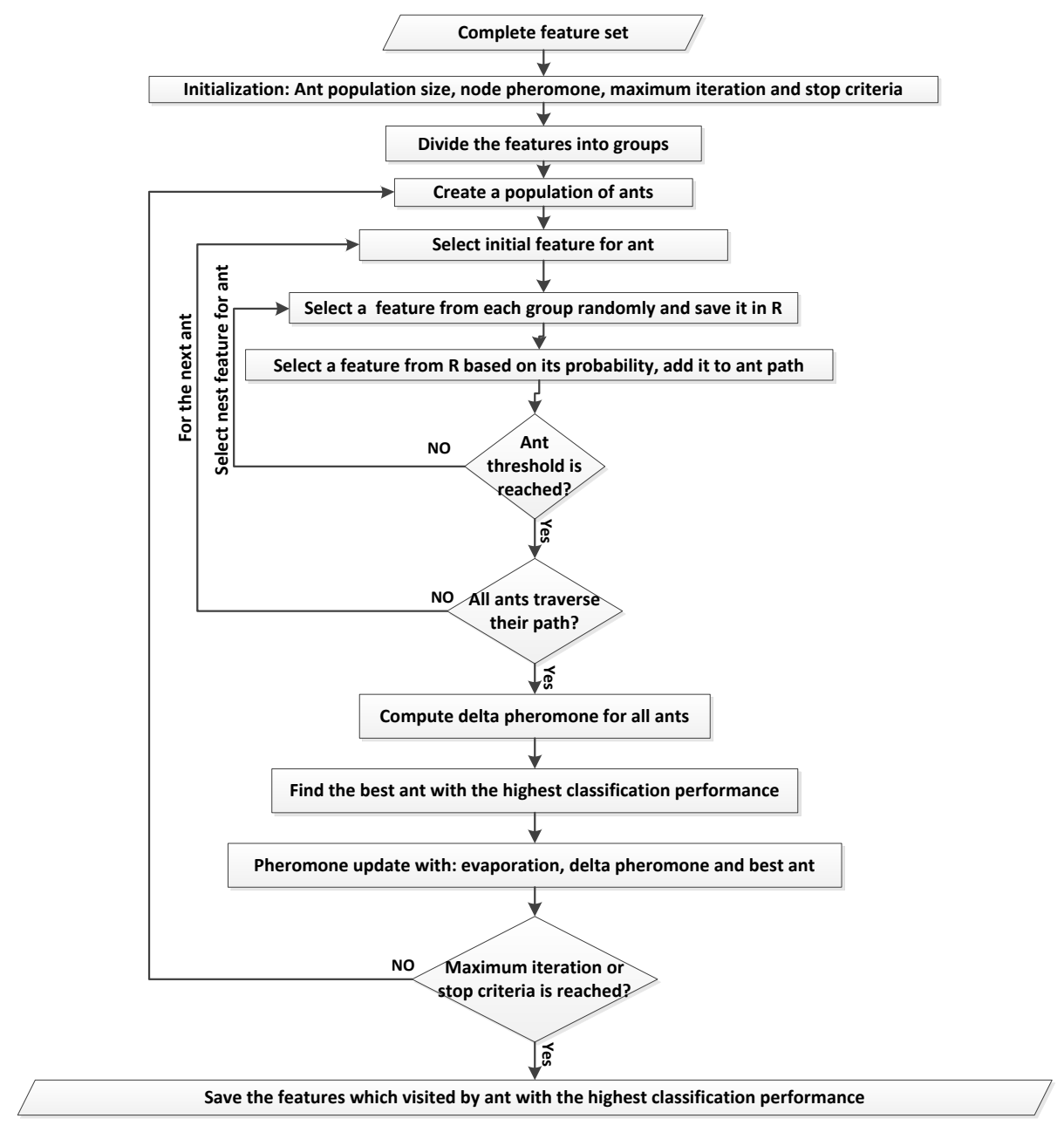

Fig 4. Proposed first feature selection method based on ACO.

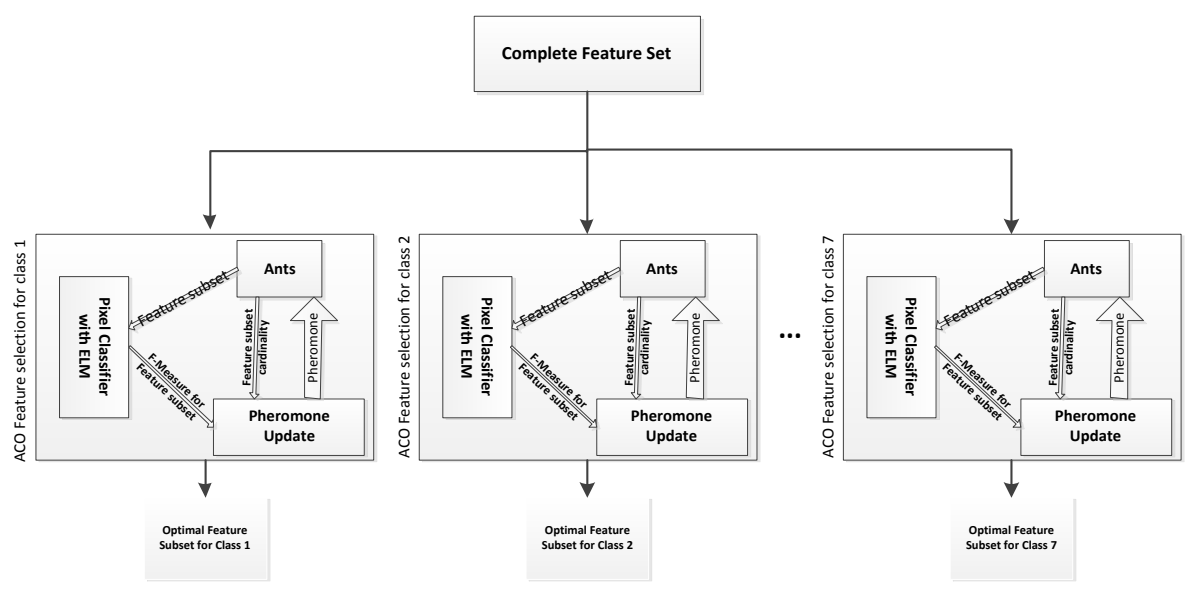

Fig. 6. Second proposed feature selection method. 
Mars Exploration Rover Spirit dataset; which has 90 images with the size of $512 \times 512$; is used as an image dataset in this research [25]. These images are the sub images of Home Plate South panorama image. Some parts of this dataset are shown in Fig. 7. Seven classes of rocks and sands are determined in [3] for the task of pixel classification which are depicted in Table 3.

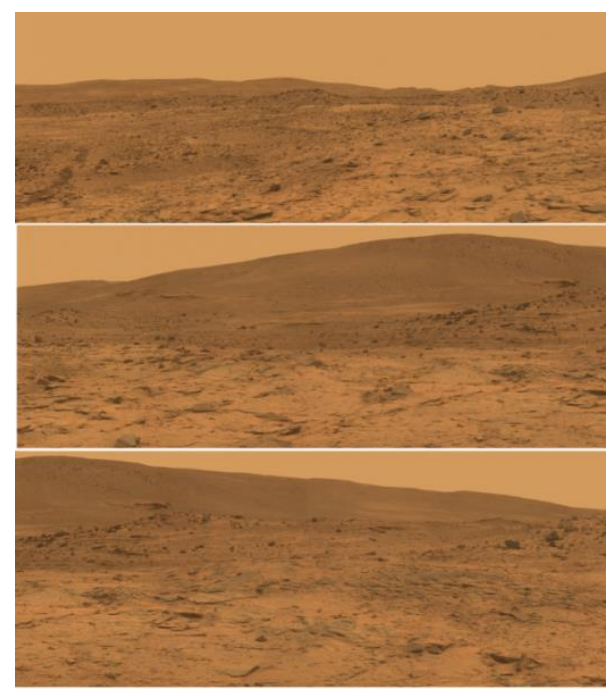

Figure.7. Some parts of Home Plate South panorama image

TABLE 3

PIXEL Classes.

\begin{tabular}{|c|c|c|c|c|c|c|c|}
\hline $\begin{array}{c}\text { CLASS } \\
\text { SYMBO } \\
\text { L }\end{array}$ & C1 (ROVER TRACKS) & $\begin{array}{l}\text { C2 (SMALL BLACK } \\
\text { STONE AND SAND) }\end{array}$ & $\begin{array}{c}\text { C3 (MEDIUM } \\
\text { BLACK STONE AND } \\
\text { SAND) }\end{array}$ & $\begin{array}{c}\text { C4 (LAYERED } \\
\text { ROCKS) }\end{array}$ & $\begin{array}{l}\text { C5 (WAVE } \\
\text { ROCKS) }\end{array}$ & $\begin{array}{c}\text { C6(DARK } \\
\text { LARGE SIZE } \\
\text { ROCKS WITH } \\
\text { SHADOW) }\end{array}$ & $\begin{array}{l}\text { C7 (FLAT } \\
\text { ROCKS) }\end{array}$ \\
\hline $\begin{array}{l}\text { IMAGE } \\
\text { SAMPLE }\end{array}$ & & & & & & & \\
\hline & & & & & & & \\
\hline
\end{tabular}

To show the utility of the proposed feature selection algorithms a series of experiments are conducted. We implement the proposed algorithms on a machine with $2.26 \mathrm{GHz}$ Corei7 CPU and 6GB of RAM. First, the images are zero padded by 10 pixels per side. Each pixel is windowed with a $21 \times 21$ window around it. Wavelet decomposition is computed in each window for the gray image, norm of rows and columns of LH and norm of rows and columns of HL lead to 4 vectors. The mean and standard deviation of these vectors are 8 wavelet features per pixel. In color features, the number of dominant colors is set to 8 , so, 24 features are computed for R, G and B components. Also, mean and standard deviation of R, G and B components form 6 color statistic features. Finally, with 4 bins for the histogram of R, G and B, 12 features are computed. Therefore, a feature vector of dimension 50 is extracted for each pixel.

We have seven SVMs and ELMs for seven classes; each one classifies only one type of rock. To train each classifier, the pixels corresponding to each rock are considered as positive samples and the remaining pixels are considered as negative samples. Table 4 shows the number of positive and negative samples for all pixel classes used in this research. All classifiers are trained for $60 \%$ of these positive and negative pixels and the remained $40 \%$ is used as unseen data in test phase.

In these experiments, various parameter values were tested for KNNs, GA and the proposed algorithms in both feature selection and pixel classification tasks. According to our experiments, the highest performance in each method is achieved by setting the parameters to values shown in Table 5. For classification task, sequential minimization optimization (SMO) is selected as learning method in SVM with maximum iteration of 20000 and kernel cache limit of 1000. The Multilayer Perceptron (MLP) with scale [-0.01, 0.01], Gaussian kernel with $\sigma=4$ and linear kernel functions are used. 
TABLE 4

Number of Positive and Negative Samples for All Pixel Classes.

\begin{tabular}{|c|c|c|c|c|c|c|c|}
\hline & Class 1 & Class 2 & Class 3 & Class 4 & Class 5 & Class 6 & Class 7 \\
\hline Number of positive samples & 5651 & 5470 & 4941 & 10771 & 8682 & 4959 & 5475 \\
\hline Number of negative samples & 5734 & 4365 & 5412 & 12544 & 9322 & 4566 & 7165 \\
\hline Sum & 11385 & 9835 & 10353 & 23515 & 18004 & 9525 & 11640 \\
\hline
\end{tabular}

TABLE 5

PARAmeter Settings For GA AND the Proposed Algorithms.

\begin{tabular}{|c|c|c|c|c|c|c|c|c|c|c|c|c|}
\hline Methods & Iteration & Population & $\begin{array}{c}\text { Initial } \\
\text { pheromone }\end{array}$ & $\begin{array}{l}\text { Crossover } \\
\text { Probability }\end{array}$ & $\begin{array}{c}\text { Mutation } \\
\text { Probability }\end{array}$ & $\delta$ & $\gamma$ & $\alpha$ & $\beta$ & $\rho$ & $\varphi$ & $\omega$ \\
\hline GA & 100 & 100 & - & $\begin{array}{l}0.6 \\
\end{array}$ & 0.008 & - & - & - & - & - & - & \\
\hline $\begin{array}{l}\text { Proposed } \\
\text { Algorithms }\end{array}$ & 100 & 100 & 1 & - & - & 1 & 1 & 0.6 & 0.4 & 0.3 & 0.1 & 0.9 \\
\hline
\end{tabular}

\section{EXPERIMENTAL RESULTS}

Our proposed pixel classification scheme with feature selection methods are evaluated by precision, recall, Fmeasure and accuracy metrics which are computed by the Eqs. (11)-(14).

$$
\begin{aligned}
& \text { Precision }=\frac{\text { Number of corretly classified positive samples }}{\text { Number of all samples classified into positive samples }} \\
& \text { Recall }=\frac{\text { Number of corretly classified positive samples }}{\text { Number of positive samples }} \\
& \text { Fmeasure }=2 \times(\text { Precision } \times \text { Recall }) \text { Precision }+ \text { Recall } \\
& \text { Accuracy }=\frac{\text { Number of correctly classified positive and negative samples }}{\text { Number of all test samples }}
\end{aligned}
$$

The results are divided into six parts. The results of classification accuracy with complete feature set, selected features by the first and second feature selection methods are reported in the following first three parts. In part 7.4, run time comparison is discussed. Statistical comparisons are reported in part 7.5 and finally segmentation results are depicted in part 7.6.

\subsection{Proposed Pixel Classifier with Complete Feature Set}

First experiment is done for the comparison between ELM, SVM and KNNs performances for complete feature set. Table 6 shows the precision, recall, Fmeasure and accuracy for these classifiers with complete feature set. Average results in table 6 show that ELM classifier outperforms other classifiers with complete feature set. ELM classifier with RBF kernel has the best average Fmeasure and accuracy of 0.9107 and 0.9023 , respectively. It outperforms the SVM with the average Fmeasure and accuracy of

\begin{tabular}{|c|c|c|c|c|c|c|c|c|c|c|c|c|c|c|c|}
\hline & & \multicolumn{3}{|c|}{ Complete Feature set } & \multicolumn{3}{|c|}{$\begin{array}{c}\begin{array}{c}\text { Selected Features by first } \\
\text { method }\end{array} \\
\end{array}$} & \multicolumn{2}{|c|}{$\begin{array}{c}\text { Complete Feature } \\
\text { set }\end{array}$} & \multicolumn{2}{|c|}{$\begin{array}{c}\text { Selected Features by } \\
\text { first method }\end{array}$} & \multicolumn{2}{|c|}{$\begin{array}{c}\text { Complete Feature } \\
\text { set }\end{array}$} & \multicolumn{2}{|c|}{$\begin{array}{l}\text { Selected Features by } \\
\text { first method }\end{array}$} \\
\hline & & $\begin{array}{l}\text { SVM } \\
\text { MLP }\end{array}$ & $\begin{array}{l}\text { SVM } \\
\text { Linear }\end{array}$ & $\begin{array}{c}\text { SVM } \\
\text { Gaussian }\end{array}$ & $\begin{array}{l}\text { SVM } \\
\text { MLP }\end{array}$ & $\begin{array}{c}\text { SVM } \\
\text { Linear }\end{array}$ & $\begin{array}{c}\text { SVM } \\
\text { Gaussian }\end{array}$ & $\begin{array}{c}\text { KNNs(k } \\
=5)\end{array}$ & $\begin{array}{l}\text { KNNs(k } \\
=3)\end{array}$ & $\begin{array}{c}\text { KNNs(k } \\
=5)\end{array}$ & $\begin{array}{c}\text { KNNs } \\
(\mathrm{k}=3)\end{array}$ & $\begin{array}{c}\text { ELM } \\
\text { RBF }\end{array}$ & $\begin{array}{c}\text { ELM } \\
\text { Linear }\end{array}$ & $\begin{array}{l}\text { ELM } \\
\text { RBF }\end{array}$ & $\begin{array}{c}\text { ELM } \\
\text { Linear }\end{array}$ \\
\hline \multirow{4}{*}{ C1 } & Precision & 0.6139 & 0.6553 & 0.6508 & 0.6110 & 0.6706 & 0.6453 & 0.6254 & 0.6342 & 0.6043 & 0.6234 & 0.6203 & 0.8223 & 0.6187 & 0.7786 \\
\hline & Recall & 0.9874 & 0.9511 & 0.9208 & 0.9867 & 0.9039 & 0.9067 & 0.9463 & 0.9533 & 0.9421 & 0.9243 & 0.9860 & 0.8906 & 0.9832 & 0.9381 \\
\hline & Fmeasure & 0.7571 & 0.7760 & 0.7626 & 0.7546 & 0.7700 & 0.7540 & 0.7530 & 0.7616 & 0.7363 & 0.7447 & 0.7615 & 0.8551 & 0.7594 & 0.8509 \\
\hline & Accuracy & 0.6855 & 0.7274 & 0.8536 & 0.6816 & 0.7319 & 0.8197 & 0.6920 & 0.7039 & 0.6651 & 0.6853 & 0.6935 & 0.8502 & 0.6909 & 0.8369 \\
\hline \multirow{4}{*}{$\mathrm{C} 2$} & Precision & 0.9975 & 0.9991 & 0.9978 & 0.9978 & 0.9989 & 0.9876 & 0.9535 & 0.9632 & 0.9418 & 0.9612 & 0.9637 & 0.9658 & 0.8914 & 0.9611 \\
\hline & Recall & 0.8600 & 0.8348 & 0.8603 & 0.8526 & 0.8328 & 0.8633 & 0.8245 & 0.8043 & 0.8175 & 0.7944 & 0.9766 & 0.9427 & 0.9872 & 0.8613 \\
\hline & Fmeasure & 0.9237 & 0.9096 & 0.9240 & 0.9195 & 0.9083 & 0.9213 & 0.8843 & 0.8766 & 0.8752 & 0.8698 & 0.9701 & 0.9541 & 0.9368 & 0.9084 \\
\hline & Accuracy & 0.9209 & 0.9077 & 0.8750 & 0.9170 & 0.9065 & 0.8710 & 0.8800 & 0.8741 & 0.8704 & 0.8678 & 0.9665 & 0.9496 & 0.9260 & 0.9035 \\
\hline \multirow{4}{*}{$\mathrm{C3}$} & Precision & 0.9952 & 0.9981 & 0.9976 & 0.9791 & 0.9981 & 0.9765 & 0.9032 & 0.9243 & 0.9008 & 0.9150 & 0.9421 & 0.9918 & 0.9931 & 0.8518 \\
\hline & Recall & 0.7520 & 0.6800 & 0.7363 & 0.7770 & 0.6811 & 0.7098 & 0.7357 & 0.7466 & 0.7380 & 0.7281 & 0.9458 & 0.7942 & 0.7993 & 0.8369 \\
\hline & Fmeasure & 0.8567 & 0.8089 & 0.8473 & 0.8664 & 0.8097 & 0.8221 & 0.8108 & 0.8260 & 0.8113 & 0.8109 & 0.9439 & 0.8820 & 0.8857 & 0.8442 \\
\hline & Accuracy & 0.8799 & 0.8467 & 0.7922 & 0.8857 & 0.8472 & 0.7849 & 0.8362 & 0.8499 & 0.8362 & 0.8380 & 0.9464 & 0.8986 & 0.9016 & 0.8527 \\
\hline \multirow{4}{*}{ C4 } & Precision & 0.9062 & 0.9436 & 0.8971 & 0.8937 & 0.9501 & 0.8876 & 0.8622 & 0.8865 & 0.8431 & 0.8734 & 0.9291 & 0.9209 & 0.9143 & 0.9642 \\
\hline & Recall & 0.9440 & 0.9174 & 0.9516 & 0.9541 & 0.9136 & 0.9312 & 0.9233 & 0.9543 & 0.8876 & 0.9354 & 0.9414 & 0.9180 & 0.9498 & 0.9231 \\
\hline & Fmeasure & 0.9247 & 0.9303 & 0.9236 & 0.9229 & 0.9315 & 0.9089 & 0.8917 & 0.9191 & 0.8647 & 0.9033 & 0.9352 & 0.9194 & 0.9317 & 0.9432 \\
\hline & Accuracy & 0.9290 & 0.9365 & 0.8906 & 0.9264 & 0.9379 & 0.8788 & 0.8964 & 0.9225 & 0.8718 & 0.9075 & $\mathbf{0 . 9 3 9 7}$ & 0.9257 & 0.9357 & 0.9486 \\
\hline
\end{tabular}
0.8726 and 0.8670 , respectively and KNNs with 0.8463 and 0.8439 .

TABLE 6

Classification ACCuracy for COMPLete and Optimal Feature SET With SVM, ELM and KNNs. 


\begin{tabular}{|c|c|c|c|c|c|c|c|c|c|c|c|c|c|c|c|}
\hline \multirow{4}{*}{ C5 } & Precision & 0.8705 & 0.8524 & 0.8965 & 0.8812 & 0.8532 & 0.8931 & 0.8565 & 0.8578 & 0.8523 & 0.8412 & 0.9178 & 0.8302 & 0.9107 & 0.8743 \\
\hline & Recall & 0.9357 & 0.9423 & 0.8942 & 0.9100 & 0.9301 & 0.8879 & 0.9133 & 0.9104 & 0.8831 & 0.9097 & 0.9034 & 0.9929 & 0.8991 & 0.9315 \\
\hline & Fmeasure & 0.9020 & 0.8951 & 0.8953 & 0.8954 & 0.8900 & 0.8905 & 0.8832 & 0.8833 & 0.8674 & 0.8741 & 0.9105 & 0.9043 & 0.9048 & 0.9019 \\
\hline & Accuracy & 0.9019 & 0.8935 & 0.8697 & 0.8974 & 0.8891 & 0.8602 & 0.8844 & 0.8840 & 0.8698 & 0.8736 & 0.9144 & 0.8986 & 0.9088 & 0.9024 \\
\hline \multirow{4}{*}{ C6 } & Precision & 0.8080 & 0.9373 & 0.8024 & 0.7082 & 0.9017 & 0.7937 & 0.7976 & 0.9155 & 0.7732 & 0.9015 & 0.8985 & 0.8951 & 0.8682 & 0.8514 \\
\hline & Recall & 0.9020 & 0.5824 & 0.8843 & 0.8543 & 0.5647 & 0.8518 & 0.9031 & 0.6534 & 0.8942 & 0.6016 & 0.9060 & 0.7893 & 0.8725 & 0.7654 \\
\hline & Fmeasure & 0.8524 & 0.7184 & 0.8413 & 0.7744 & 0.6945 & 0.8217 & 0.8470 & 0.7625 & 0.8293 & 0.7216 & 0.9022 & 0.8388 & 0.8703 & 0.8061 \\
\hline & Accuracy & 0.8374 & 0.7623 & 0.9013 & 0.7409 & 0.7413 & 0.8619 & 0.8302 & 0.7882 & 0.8084 & 0.7584 & 0.8978 & 0.8421 & 0.8647 & 0.8083 \\
\hline \multirow{4}{*}{ C7 } & Precision & 0.9936 & 0.9400 & 0.9989 & 0.9926 & 0.9178 & 0.9776 & 0.9878 & 0.9900 & 0.9653 & 0.9742 & 0.9448 & 0.9704 & 0.9989 & 0.8456 \\
\hline & Recall & 0.8087 & 0.8537 & 0.6727 & 0.8107 & 0.8494 & 0.6856 & 0.7524 & 0.7764 & 0.7442 & 0.7601 & 0.9591 & 0.8011 & 0.8454 & 0.9302 \\
\hline & Fmeasure & 0.8917 & 0.8948 & 0.8040 & 0.8925 & 0.8822 & 0.8060 & 0.8541 & 0.8702 & 0.8404 & 0.8539 & 0.9519 & 0.8776 & 0.9157 & 0.8858 \\
\hline & Accuracy & 0.9149 & 0.9130 & 0.8981 & 0.9154 & 0.9018 & 0.8668 & 0.8887 & 0.8998 & 0.8776 & 0.8874 & 0.9580 & 0.9033 & 0.9326 & 0.8962 \\
\hline \multicolumn{2}{|c|}{ Average Precision } & 0.8835 & 0.9036 & 0.8915 & 0.8662 & 0.8986 & 0.8802 & 0.8551 & 0.8816 & 0.8401 & 0.8699 & 0.8880 & 0.9137 & 0.8850 & 0.8467 \\
\hline \multicolumn{2}{|c|}{ Average Recall } & 0.8842 & 0.8231 & 0.8457 & 0.8779 & 0.8108 & 0.8337 & 0.8569 & 0.8283 & 0.8438 & 0.8076 & 0.9454 & 0.8755 & 0.8952 & 0.8837 \\
\hline \multicolumn{2}{|c|}{$\begin{array}{c}\text { Average } \\
\text { F-measure } \\
\end{array}$} & 0.8726 & 0.8475 & 0.8568 & 0.8608 & 0.8408 & 0.8463 & 0.8463 & 0.8427 & 0.8320 & 0.8254 & 0.9107 & 0.8901 & 0.8802 & 0.8579 \\
\hline \multicolumn{2}{|c|}{$\begin{array}{l}\text { Average } \\
\text { Accuracy }\end{array}$} & 0.8670 & 0.8553 & 0.8686 & 0.8520 & 0.8508 & 0.8490 & 0.8439 & 0.8460 & 0.8284 & 0.8311 & 0.9023 & 0.8954 & 0.8800 & 0.8783 \\
\hline \multicolumn{4}{|c|}{\begin{tabular}{|c|}
$\begin{array}{c}\text { Optimal feature subset found by first } \\
\text { proposed method and SVM }\end{array}$ \\
\end{tabular}} & \multicolumn{12}{|c|}{ LH_meanL, HL_stdH, HL_stdL, DCDR1, DCDR5, DCDG4, DCDB1, Bm, Bs, R1, R6, G2,G3, B1,B4,B6,B7 } \\
\hline \multicolumn{4}{|c|}{$\begin{array}{c}\text { Optimal feature subset found by first } \\
\text { proposed method and KNNs }\end{array}$} & \multicolumn{12}{|c|}{ LH_stdL, HL_meanL, HL_stdL, DCDR1, DCDR7, DCDG1, DCDB1,DCDB3, Bm,Bs,R1 ,R6, G2,G3, G4,B1,B6,B8 } \\
\hline \multicolumn{4}{|c|}{$\begin{array}{l}\text { Optimal feature subset found by first } \\
\text { proposed method and ELM classifier }\end{array}$} & \multicolumn{12}{|c|}{ HL_stdH, HL_stdL, DCDR4, DCDR8, DCDG5, DCDB1, DCDB5, DCDB7, Bs, R4, G8, B2, B6 } \\
\hline
\end{tabular}

\subsection{First Feature Selection Method}

As it was mentioned before, two feature selection methods were proposed in this research. The last 3 rows of table 6 show that the first proposed method selects 13, 17 and 18 features for ELM, SVM and KNNs classifiers, respectively. From these results, it can be concluded that after dimension reduction by the first proposed method, the classification performance is preserved with a little decrease. However, we'll show later that the main advantage of the first feature selection method is that it decreases the run time of classifiers in both train and test phases considerably. Also, there is a difference in optimal feature subsets for a system with ELM, SVM and KNNs, which stems from differences in performance of subsets in different classifiers; this leads ants to select different paths and consequently different features.

It is shown that how the first feature selection method is in correlation with the features efficiencies in classification performance. For this task, features are first sorted based on their pheromone. Then, the classification accuracies are computed for first ranked feature, two top ranked features, three top ranked features and so on. These accuracies are depicted in Fig. 8 for ELM, SVM and KNNs in their best parameters as RBF kernel, MLP kernel and k=5, respectively. For ELM, SVM and KNNs classifiers, after respectively, 13, 17 and 18 top ranked features, accuracies do not increase any more. This means that our first proposed feature selection algorithm converged correctly in 13,17 and 18 features for these classifiers. 


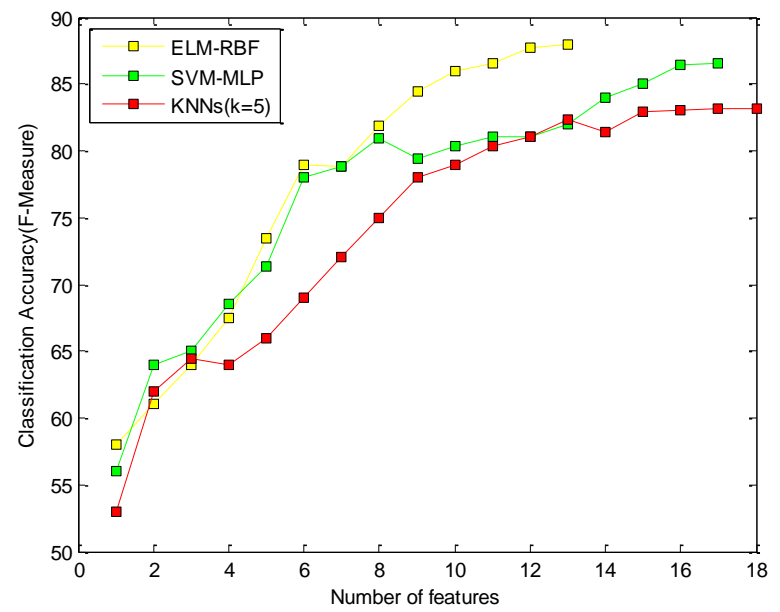

Fig. 8. Classification accuracies for highly ranked feature subsets.

\subsection{Second Feature Selection Method}

The second proposed feature selection method provides an optimal feature subset for each pixel classifier separately. For the majority of classifiers such as KNNs this task is not applicable, since only one model is trained for all classes and it is not possible to introduce different feature subsets for each class. In the proposed scheme, since each class has its own classifier as either ELM or SVM, it is possible to train each classifier by its own feature subset. Optimal feature subsets and reduction percents are presented in table 7. The lowest cardinality is appeared for the selected features for class C5 by ELM classifier with RBF kernel which selects 12 features among all 50 features.

The second feature selection method decreases the dimension of the feature set and increases the classification accuracy for the majority of pixel classes. Precision, recall, Fmeasure and accuracy of the second proposed method are reported for ELM and SVM classifiers with their different kernels in Table 8. This table also shows that how performances are affected by four feature sets including complete feature set, selected features by the first and second method and selected features by genetic algorithm. The second proposed feature selection method outperforms the first proposed feature selection method and genetic algorithmbased feature selection. Furthermore, the second proposed algorithm slightly outperforms the classification performance of classifiers with complete feature set. Therefore, the second feature selection method not only decreases the feature set cardinality but also slightly increases the classification accuracy.

TABLE 7

OPTIMAL FeATURe SubSETS FOR EACH Pixel Class.

\begin{tabular}{|c|c|c|c|c|c|c|c|c|}
\hline & \multicolumn{2}{|c|}{ ELM_RBF } & \multicolumn{2}{c|}{ ELM_Linear } & \multicolumn{2}{c|}{ SVM_MLP } & \multicolumn{2}{c|}{ SVM_Linear } \\
\hline & $\begin{array}{c}\text { Selected } \\
\text { Features }\end{array}$ & $\begin{array}{c}\text { Reduction } \\
\text { Percent }\end{array}$ & $\begin{array}{c}\text { Selected } \\
\text { Features }\end{array}$ & $\begin{array}{c}\text { Reduction } \\
\text { Percent }\end{array}$ & $\begin{array}{c}\text { Selected } \\
\text { Features }\end{array}$ & $\begin{array}{c}\text { Reduction } \\
\text { Percent }\end{array}$ & $\begin{array}{c}\text { Selected } \\
\text { Features }\end{array}$ & $\begin{array}{c}\text { Reduction } \\
\text { Percent }\end{array}$ \\
\hline C1 & 14 & $72 \%$ & 13 & $74 \%$ & 16 & $68 \%$ & 17 & $66 \%$ \\
\hline C2 & 13 & $74 \%$ & 15 & $70 \%$ & 19 & $62 \%$ & 19 & $62 \%$ \\
\hline C3 & 16 & $68 \%$ & 17 & $66 \%$ & 17 & $66 \%$ & 18 & $64 \%$ \\
\hline C4 & 13 & $74 \%$ & 14 & $72 \%$ & 19 & $62 \%$ & 21 & $58 \%$ \\
\hline C5 & 12 & $76 \%$ & 20 & $60 \%$ & 21 & $58 \%$ & 20 & $60 \%$ \\
\hline C6 & 14 & $72 \%$ & 19 & $62 \%$ & 15 & $70 \%$ & 17 & $66 \%$ \\
\hline
\end{tabular}

TABLE 8

Classification ACCURACy FOR SeCONd Proposed Method and GA.

\begin{tabular}{|c|c|c|c|c|c|c|c|c|c|}
\hline & & \multicolumn{2}{|c|}{ Complete Feature set } & \multicolumn{2}{|c|}{$\begin{array}{c}\text { Selected Features by } \\
\text { first method }\end{array}$} & \multicolumn{2}{|c|}{$\begin{array}{l}\text { Selected Features by } \\
\text { second method }\end{array}$} & \multicolumn{2}{|c|}{ Selected Features by GA } \\
\hline & & SVM_MLP & ELM_RBF & SVM_MLP & ELM_RBF & SVM_MLP & ELM_RBF & SVM_MLP & ELM_RBF \\
\hline \multirow{4}{*}{ C1 } & Precision & 0.6139 & 0.6203 & 0.6110 & 0.6187 & 0.5889 & 0.6219 & 0.6122 & 0.6054 \\
\hline & Recall & 0.9874 & 0.9860 & 0.9867 & 0.9832 & 0.9892 & 0.9785 & 0.9619 & 0.9614 \\
\hline & Fmeasure & 0.7571 & 0.7615 & 0.7546 & 0.7594 & 0.7390 & 0.7604 & 0.7482 & 0.7429 \\
\hline & Accuracy & 0.6855 & 0.6935 & 0.6816 & 0.6909 & 0.6519 & 0.6940 & 0.6787 & 0.6698 \\
\hline \multirow{5}{*}{$\mathrm{C2}$} & Precision & 0.9975 & 0.9637 & 0.9978 & 0.8914 & 0.9960 & 0.9575 & 0.9935 & 0.8854 \\
\hline & Recall & 0.8600 & 0.9766 & 0.8526 & 0.9872 & 0.8883 & 0.9940 & 0.8415 & 0.9743 \\
\hline & Fmeasure & 0.9237 & 0.9701 & 0.9195 & 0.9368 & 0.9391 & 0.9754 & 0.9112 & 0.9277 \\
\hline & Accuracy & 0.9209 & 0.9665 & 0.9170 & 0.9260 & 0.9359 & 0.9721 & 0.9088 & 0.9156 \\
\hline & Precision & 0.9952 & 0.9421 & 0.9791 & 0.9931 & 0.9953 & 0.9923 & 0.9713 & 0.9834 \\
\hline
\end{tabular}




\begin{tabular}{|c|c|c|c|c|c|c|c|c|c|}
\hline \multirow[t]{3}{*}{ C3 } & Recall & 0.7520 & 0.9458 & 0.7770 & 0.7993 & 0.7779 & 0.9443 & 0.7455 & 0.7923 \\
\hline & Fmeasure & 0.8567 & 0.9439 & 0.8664 & $\mathbf{0 . 8 8 5 7}$ & 0.8732 & 0.9677 & 0.8436 & 0.8775 \\
\hline & Accuracy & 0.8799 & 0.9464 & 0.8857 & 0.9016 & 0.8922 & 0.9699 & 0.8680 & 0.8945 \\
\hline \multirow{4}{*}{ C4 } & Precision & 0.9062 & 0.9291 & 0.8937 & 0.9143 & 0.9077 & 0.9396 & 0.8965 & 0.9145 \\
\hline & Recall & 0.9440 & 0.9414 & 0.9541 & 0.9498 & 0.9517 & 0.9714 & 0.9576 & 0.9321 \\
\hline & Fmeasure & 0.9247 & 0.9352 & 0.9229 & 0.9317 & 0.9293 & 0.9552 & 0.9260 & 0.9232 \\
\hline & Accuracy & 0.9290 & 0.9397 & 0.9264 & 0.9357 & 0.9330 & 0.9579 & 0.9293 & 0.9284 \\
\hline \multirow{4}{*}{ C5 } & Precision & 0.8705 & 0.9178 & 0.8812 & 0.9107 & 0.8945 & 0.9587 & 0.8633 & 0.8954 \\
\hline & Recall & 0.9357 & 0.9034 & 0.9100 & 0.8991 & 0.9317 & 0.9111 & 0.9042 & 0.8933 \\
\hline & Fmeasure & 0.9020 & 0.9105 & 0.8954 & 0.9048 & 0.9127 & 0.9342 & 0.8833 & 0.8943 \\
\hline & Accuracy & 0.9019 & 0.9144 & 0.8974 & 0.9088 & 0.9141 & 0.9382 & 0.8848 & 0.8982 \\
\hline \multirow{4}{*}{ C6 } & Precision & 0.8080 & 0.8985 & 0.7082 & 0.8682 & 0.8037 & 0.8854 & 0.7854 & 0.8546 \\
\hline & Recall & 0.9020 & 0.9060 & 0.8543 & 0.8725 & 0.8992 & 0.8986 & 0.8945 & 0.8632 \\
\hline & Fmeasure & 0.8524 & 0.9022 & 0.7744 & 0.8703 & 0.8487 & 0.8919 & 0.8364 & $\mathbf{0 . 8 5 8 8}$ \\
\hline & Accuracy & 0.8374 & 0.8978 & 0.7409 & 0.8647 & $\mathbf{0 . 8 3 3 2}$ & 0.8867 & 0.8178 & 0.8523 \\
\hline \multirow{4}{*}{ C7 } & Precision & 0.9936 & 0.9448 & 0.9926 & 0.9989 & 0.9863 & 0.9912 & 0.9932 & 0.9738 \\
\hline & Recall & 0.8087 & 0.9591 & 0.8107 & 0.8454 & 0.8247 & 0.8965 & 0.7913 & 0.8055 \\
\hline & Fmeasure & $\mathbf{0 . 8 9 1 7}$ & 0.9519 & $\mathbf{0 . 8 9 2 5}$ & 0.9157 & $\mathbf{0 . 8 9 8 3}$ & 0.9415 & 0.8808 & 0.8816 \\
\hline & Accuracy & 0.9149 & 0.9580 & 0.9154 & 0.9326 & 0.9191 & 0.9517 & 0.9073 & 0.9064 \\
\hline \multicolumn{2}{|c|}{ Average Precision } & $\mathbf{0 . 8 8 3 5}$ & $\mathbf{0 . 8 8 8 0}$ & 0.8662 & 0.8850 & $\mathbf{0 . 8 8 1 7}$ & 0.9066 & 0.8736 & 0.8732 \\
\hline \multicolumn{2}{|c|}{ Average Recall } & 0.8842 & 0.9454 & 0.8779 & 0.8952 & 0.8946 & 0.9406 & 0.8709 & 0.8888 \\
\hline \multicolumn{2}{|c|}{ Average F-measure } & 0.8726 & 0.9107 & 0.8608 & 0.8802 & 0.8771 & 0.9172 & 0.8613 & 0.8722 \\
\hline \multicolumn{2}{|c|}{ Average Accuracy } & 0.8670 & 0.9023 & $\mathbf{0 . 8 5 2 0}$ & $\mathbf{0 . 8 8 0 0}$ & 0.8684 & 0.9100 & 0.8563 & 0.8664 \\
\hline
\end{tabular}

\subsection{Run Time Comparison}

Run time is so important especially for the on-board applications of Mars image segmentation. Here, the run time is considered for both train and test phases. Table 9 reports the run time of train and test phases in three classifiers with complete feature set for different parameters. Although KNN has no training time, its classification accuracy is very lower than SVM and ELM. Generally, ELM with linear kernel has the lowest run time with an average of $4402 \mathrm{~ms}$ and $180 \mathrm{~ms}$ for train and test phases respectively.

The first proposed feature selection algorithm selects the most relevant features for all classes simultaneously. Train and test samples of all classes are involved in steps 4, 5 and 7 of this algorithm while in the second feature selection algorithm the train and test samples of only one class are involved at the same time. The run times of the first and second feature selection algorithms are 1286 and 353 seconds, respectively. Note that 353 seconds is only for one class in the second feature selection method. Feature selection for other classes can be run with parallel procedures. These run times are the average run times of using different classifiers in feature selection process. It should be noted that the proposed feature selection algorithms are done once in offline phase and then selected features will be used for the train and test phases of classifiers. Therefore, their run times are not the concern for pixel classification application. The main advantage of the proposed feature selection methods is dimension reduction which decreases the run time considerably. Therefore, we also have reported the run time of classifiers with selected features by two proposed feature selection algorithms in train and test phases in Fig. 9 and Fig. 10, respectively. For these two figures some abbreviations are used for each bar. For example, 'SVM-Linear-Comp' means that SVM classifier with linear kernel is used for complete feature set and 'SVM-Linear-First' means that SVM classifier with linear kernel is used for selected features by the first proposed method. For train run times, only ELM and SVM are compared since KNNs has no train run time. It is concluded from these figures that classifiers with selected features by feature selection methods have lower run times in train and test phases. Another conclusion is that each classifier with selected features from the first and second feature selection methods has the same run time. However, the main advantage of the second proposed method is its higher accuracy which was reported in Table 8 in more details.

TABLE 9

RUN TIME OF SVM, KNN AND ELM FOR COMPLETE FEATURE SET.

\begin{tabular}{|c|c|c|c|c|c|c|c|}
\hline \multicolumn{2}{|c|}{} & ELM_RBF & ELM_Linear & SVM_MLP & SVM_Linear & KNNs(k=3) & KNNs(k=5) \\
\hline \multirow{2}{*}{ C1 } & Train time(ms $\left.{ }^{2}\right)$ & 2734 & 2032 & 7554 & 20041 & - & - \\
\cline { 2 - 8 } & Test time(ms) & 719 & 106 & 368 & 125 & 5228 & 7242 \\
\hline \multirow{2}{*}{ C2 } & Train time(ms) & 1677 & 1172 & 2471 & 1233 & - & - \\
\cline { 2 - 8 } & Test time(ms) & 463 & 72 & 133 & 46 & 4338 & 4845 \\
\hline \multirow{2}{*}{ C3 } & Train time(ms) & 2244 & 1562 & 6792 & 11011 & - & - \\
\cline { 2 - 8 } & Test time(ms) & 610 & 89 & 316 & 106 & 6876 & 7929 \\
\hline \multirow{2}{*}{ C4 } & Train time(ms) & 18318 & 12954 & 25054 & 32767 & - & - \\
\cline { 2 - 8 } & Test time(ms) & 4150 & 442 & 1214 & 432 & 26545 & 29415 \\
\hline
\end{tabular}

\footnotetext{
${ }^{2}$ The time unit for algorithms run time is millisecond with the ' $m s$ ' abbreviation.
} 


\begin{tabular}{|c|c|c|c|c|c|c|c|}
\hline C5 & Train time $(\mathrm{ms})$ & 11588 & 9046 & 17350 & 29637 & - & - \\
\cline { 2 - 8 } & Test time $(\mathrm{ms})$ & 2333 & 320 & 811 & 352 & 18337 & 20980 \\
\hline \multirow{2}{*}{ C6 } & Train time(ms) & 1882 & 1254 & 5594 & 6517 & - & - \\
\cline { 2 - 8 } & Test time $(\mathrm{ms})$ & 519 & 88 & 291 & 98 & 5842 & 6835 \\
\hline \multirow{2}{*}{ C7 } & Train time(ms) & 4608 & 2795 & 9603 & 23052 & - & - \\
\cline { 2 - 8 } & Test time(ms) & 1204 & 147 & 509 & 166 & 11589 & 14516 \\
\hline \multicolumn{2}{|c|}{ Average Train Time } & $\mathbf{6 1 5 0}$ & $\mathbf{4 4 0 2}$ & $\mathbf{1 0 6 3 1}$ & $\mathbf{1 7 7 5 1}$ & - & - \\
\hline \multicolumn{2}{|c|}{ Average Test Time } & $\mathbf{1 4 2 8}$ & $\mathbf{1 8 0}$ & $\mathbf{5 2 0}$ & $\mathbf{1 8 9}$ & $\mathbf{1 1 2 5 0}$ & $\mathbf{1 3 1 0 8}$ \\
\hline
\end{tabular}

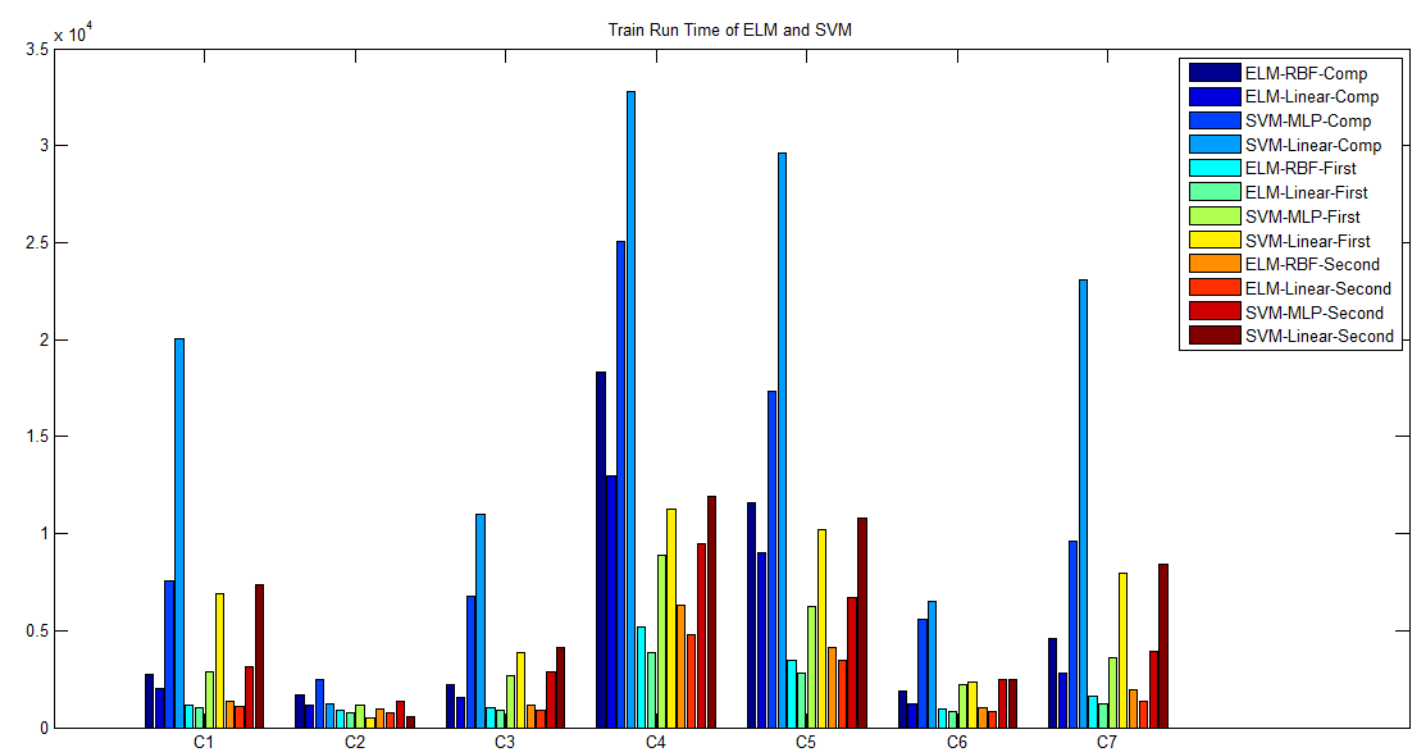

Fig. 9. Train run time for ELM and SVM classifiers with selected features of first and second feature selection methods.

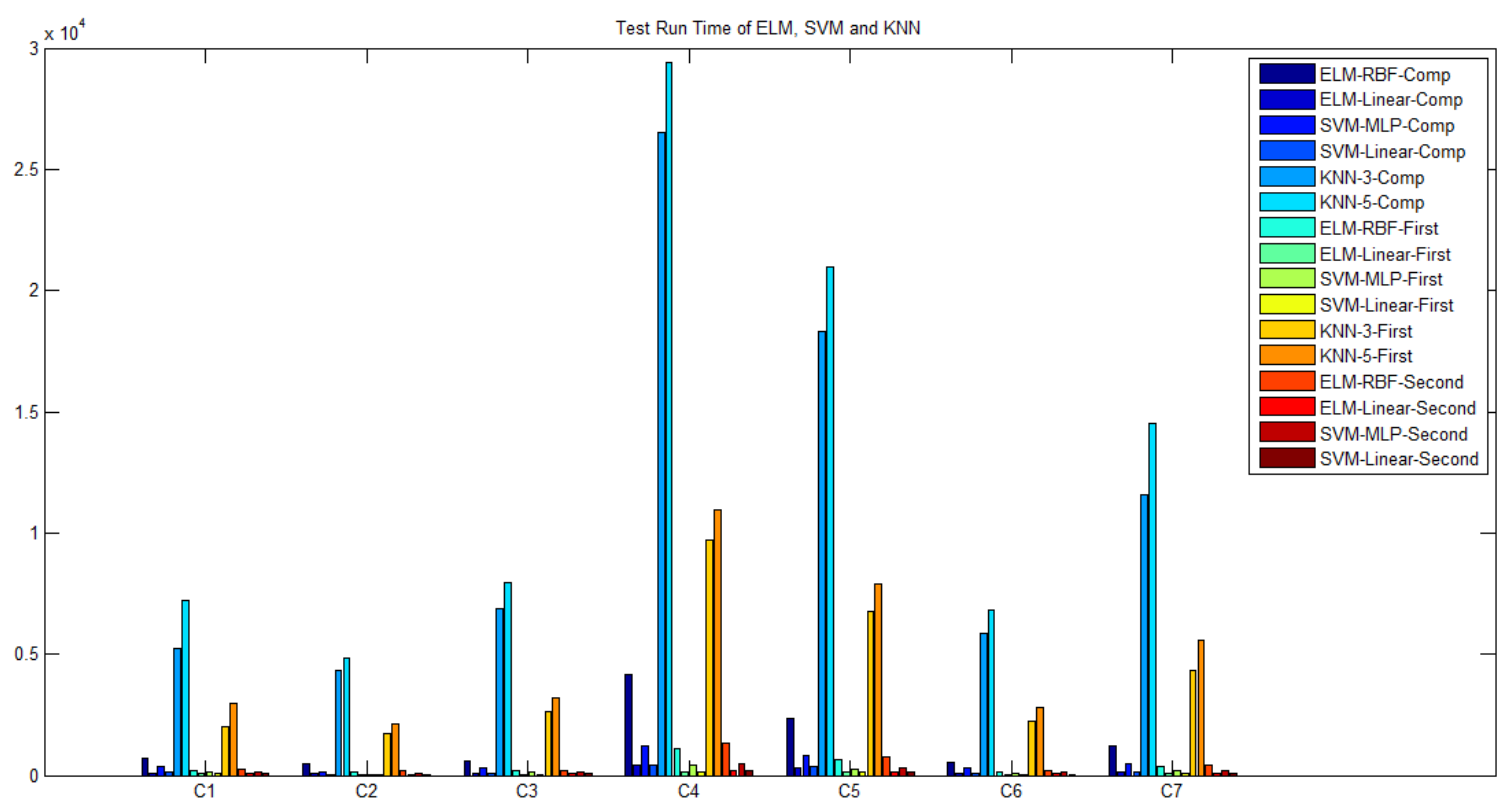

Fig. 10. Test run time for ELM, SVM and KNNs classifiers with selected features of first and second feature selection methods.

\subsection{Statistical Comparison}

Tables 6 and 8 reported the Fmeasure and accuracy of all 7 pixel classes for each method. Average Fmeasures and accuracies in all 7 classes could be used as overall performance criteria. Furthermore, a statistical test is used for the overall performance 
comparison too. In this research, for overall comparison of methods, two sets with seven elements should be compared. Therefore, parametric statistical tests aren't applicable here and pairwise U-Mann-Whitney test is used which is a non-parametric test. In this test, the null hypothesis is that two samples come from the same population against an alternative hypothesis with the meaning that the two samples are from different populations. This test provides the confidence level for the differences between sets as well as a mean rank for each set. We first determine that two sets are different with a confidence level and then determine the better set which is the set with higher mean rank. Different pairwise comparisons between classifiers with different feature sets are reported in table 10. This table shows the mean rank and confidence level between each two sets and its last column determines which set is statistically better than another. The confidence level threshold of $90 \%$ is considered. The main conclusion is that ELM with the complete feature set outperforms other classifiers statistically with a confidence level of $94 \%$. Furthermore, the classification performance of classifiers with complete feature set and with selected features are statistically the same. This means that after dimension reduction the classification performance isn't decreased statistically. Although the mean rank of classifier performances with the selected features by second feature selection method is higher than classifier performances with complete feature set, its confidence level is very low and under predefined threshold. Therefore, we reported that none of which is better than other. Finally, it is resulted that the second proposed feature selection method outperforms the genetic algorithm with the confidence level of $95.2 \%$.

TABLE 10

PAirwise Statistical COMPARISON OF All Methods.

\begin{tabular}{|c|c|c|c|c|c|}
\hline & & & $\begin{array}{l}\text { Comparative } \\
\text { Mean Ranks }\end{array}$ & Confidence Level & $\begin{array}{c}\text { Which one is better } \\
\text { statistically? }\end{array}$ \\
\hline \multirow{9}{*}{ F-Measure } & \multirow{2}{*}{$\begin{array}{l}\text { Complete } \\
\text { Feature Set }\end{array}$} & ELM and SVM & 9.57 and 5.43 & $94.4 \%$ & ELM \\
\hline & & ELM and KNNs & 10.14 and 4.84 & $98.2 \%$ & ELM \\
\hline & \multirow{3}{*}{$\begin{array}{l}\text { First Method and } \\
\text { Complete }\end{array}$} & ELM_Com and ELM_First & 9.00 and 6.00 & $82 \%$ & None \\
\hline & & SVM_Com and SVM_First & 7.86 and 7.14 & $25.1 \%$ & None \\
\hline & & KNNs_Com and KNNs_First & 8.43 and 6.57 & $59.4 \%$ & None \\
\hline & \multirow{2}{*}{$\begin{array}{l}\text { Second Method } \\
\text { and Complete }\end{array}$} & EIM_Com and ELM_Second & 7.14 and 7.86 & $25.1 \%$ & None \\
\hline & & SVM_Com and SVM_Second & 7.14 and 7.86 & $25.1 \%$ & None \\
\hline & \multirow{2}{*}{$\begin{array}{c}\text { Second Method and } \\
\text { GA }\end{array}$} & ELM_Second and ELM_GA & 9.71 and 5.29 & $95.2 \%$ & ELM_Second \\
\hline & & SVM_Second and SVM_GA & 8.43 and 6.57 & $59.4 \%$ & None \\
\hline \multirow{9}{*}{ Accuracy } & \multirow{2}{*}{\begin{tabular}{|c|} 
Complete \\
Feature Set \\
\end{tabular}} & ELM and SVM & 9.14 and 5.86 & 85.8 & None \\
\hline & & ELM and KNNs & 10.14 and 4.86 & 98.2 & ELM \\
\hline & \multirow{3}{*}{$\begin{array}{c}\text { First Method and } \\
\text { Complete }\end{array}$} & ELM_Com and ELM_First & 9 and 6 & $82 \%$ & None \\
\hline & & SVM_Com and SVM_First & 7.71 and 7.29 & $15.2 \%$ & None \\
\hline & & KNNs_Com and KNNs_First & 8.79 and 6.21 & $75 \%$ & None \\
\hline & \multirow{2}{*}{$\begin{array}{l}\text { Second Method } \\
\text { and Complete }\end{array}$} & EIM_Com and ELM_Second & 6.86 and 8.14 & $43.5 \%$ & None \\
\hline & & SVM_Com and SVM_Second & 7.14 and 7.76 & $25.1 \%$ & None \\
\hline & \multirow{2}{*}{$\begin{array}{c}\text { Second Method and } \\
\text { GA }\end{array}$} & ELM_Second and ELM_GA & 9.43 and 5.57 & $91.5 \%$ & ELM_Second \\
\hline & & SVM_Second and SVM_GA & 8.57 and 6.43 & $66.2 \%$ & None \\
\hline
\end{tabular}

\subsection{Segmentation Results}

The final results of this research are pixel classification. All seven types of rocks including Rover tracks, small black stone and sand, medium black stone and sand, layered rocks, wave rocks, dark large size rocks with shadow and flat rocks are all classified in Home Plate South sub images. However, some parts of images which were far away from Pancom are not classified to one of seven rock types. The reason is that their details are not visible. As it is clear from classified images, most errors occur in region boundaries. Since the feature vector of a pixel is derived from a window around that pixel, the pixels which are near to region boundaries are also windowed by the pixels from other classes, so they are incorrectly classified to other class labels. We assigned eight colors including red, green, blue, yellow, indigo, pink, gray and violet for classes 1-7 and non-classified parts, respectively. Fig. 11(a-c) shows some samples of classified images. 
(a)
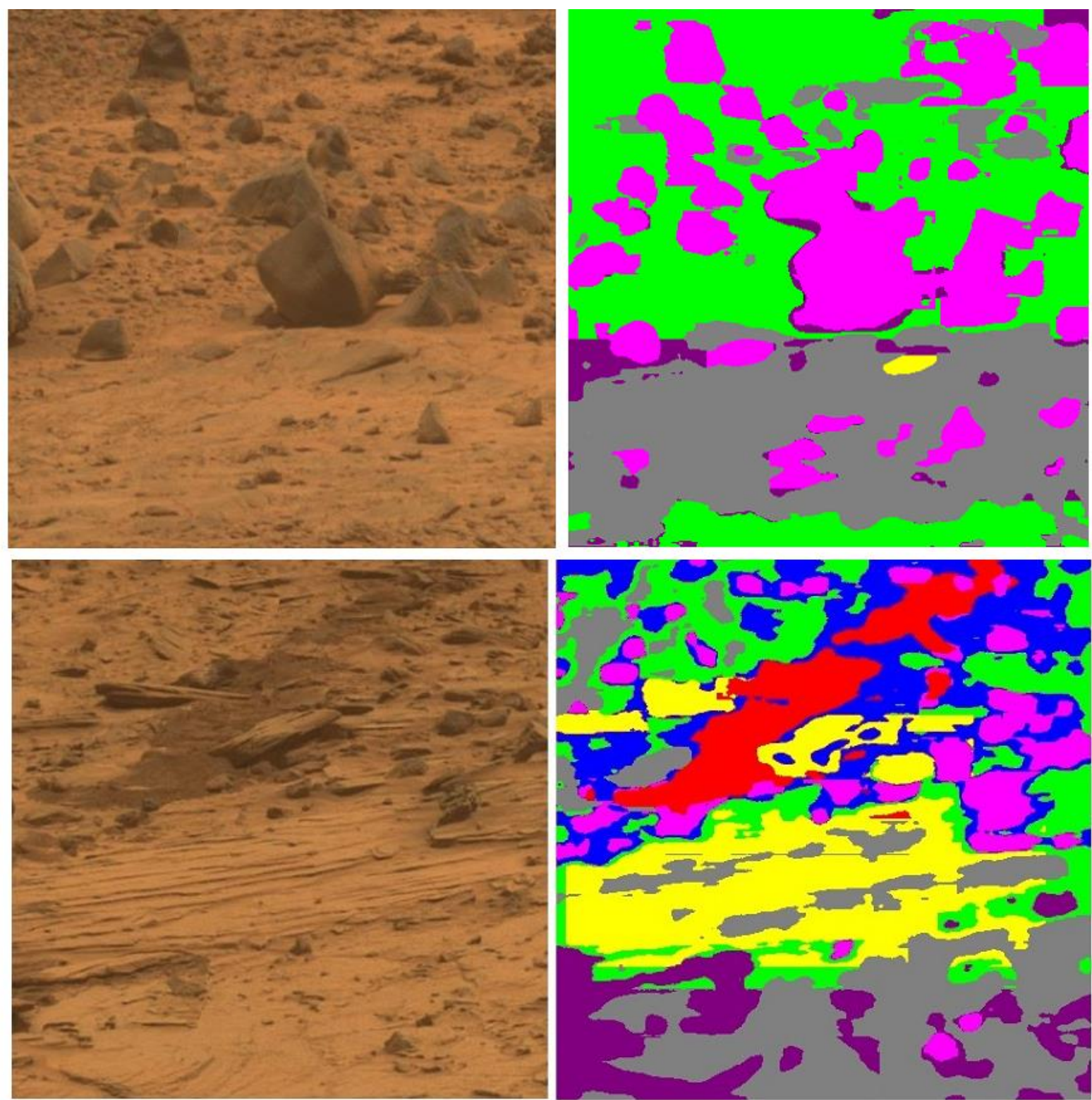

(b)

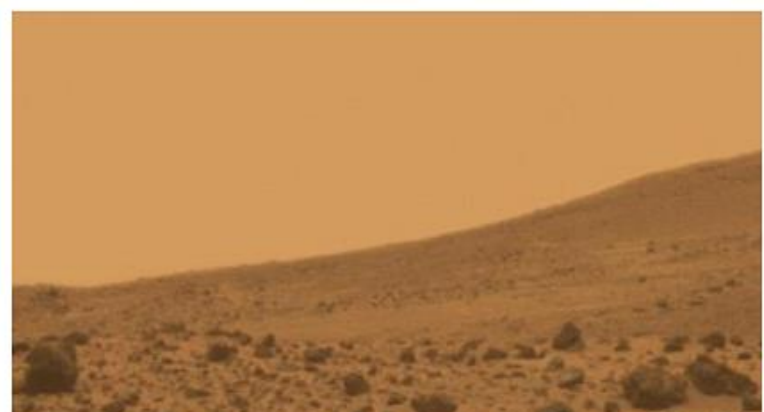

(c)

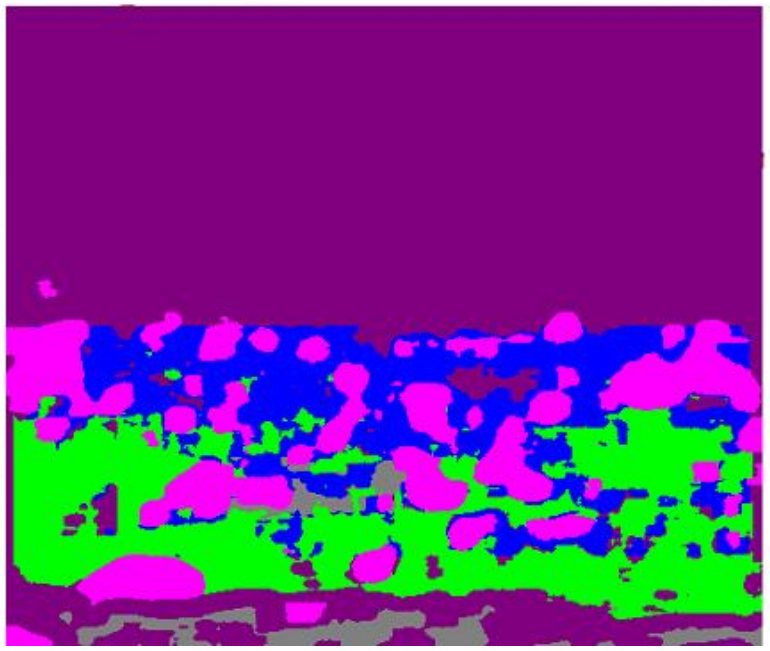

Fig. 11. Samples of classified images. 
Home plate south panorama images contain information about Mars, taken by Mars rover, a robot which explores on Mars surface. In this paper, a new pixel classification scheme was proposed which leads to Mars image segmentation. Firstly, each pixel is windowed and mapped to a feature vector including wavelet and color features. The type of extracted features was proposed by the way that all pixel classes could be discriminated from other classes and appropriate segmentation of images are achieved. Then, most relevant features among complete feature set were selected by two proposed feature selection schema based on ACO. Finally, optimal feature sets were presented for the classifiers for pixel classification. Classification of pixels segments the images into pixel groups. Our proposed model for image segmentation was compared with other methods; results showed that the proposed pixel classification scheme with complete feature set and ELM classifier outperforms other classifiers include SVM and KNNs in both accuracy and run time. Furthermore, the first feature selection method presents a feature subset which decreases the run times and preserves the accuracies of not only ELM classifier but also SVM and KNNs classifiers. Finally, the second proposed feature selection method selects an optimal feature subset for each pixel class separately. This method decreases the run time and increases the accuracies of most of classifiers simultaneously. It also outperforms the first proposed feature selection method and genetic algorithm-based feature selection. For future works, the performance of the proposed approaches can be evaluated by taking into account other classifiers such as artificial neural networks and decision trees. Other feature selection methods can be improved and applied to such systems. In addition, intrinsic property of data such as Relief weights can be used in population-based techniques such as ACO, GA and Particle swarm optimization (PSO) algorithms to increase convergence speed. Finally, other features like shape and texture features and color features in other color systems can also be applied for pixel classification task.

\section{REFERENCES}

[1] Xue, Yansong, Shuanggen Jin, and Yi Yang. "Mineral components at Martian Gale region from Mars Reconnaissance Orbiter CRISM images."

[2] Castano, R., Judd, M., Estlin, T., Anderson, R. C., Gaines, D., Castaño, A., ... \& Wagstaff, K. (2005). Current results from a rover science data analysis system. In Aerospace Conference, 2005 IEEE (pp. 356-365). IEEE.

[3] Shang, C., \& Barnes, D. (2013). Fuzzy-rough feature selection aided support vector machines for mars image classification. Computer Vision and Image Understanding, 117(3), 202-213.

[4] Pang, Y., Zhu, H., Li, X., \& Pan, J. (2016). Motion Blur Detection with an Indicator Function for Surveillance Machines, vol. 63, no. 9, pp. 5592-5601.

[5] Pang, Y., Zhu, H., Li, X., \& Li, X. (2016). Classifying discriminative features for blur detection, vol. 46, no. 10, pp. 22202227.

[6] De Cooman, G. (2005). A behavioural model for vague probability assessments. Fuzzy sets and systems, 154(3), 305-358.

[7] Huang, K., \& Aviyente, S. (2008). Wavelet feature selection for image classification. Image Processing, IEEE Transactions on, 17(9), 1709-1720.

[8] Kachanubal, T., \& Udomhunsakul, S. (2008). Rock textures classification based on textural and spectral features. Int J Comput Intell, 4, 240-246.

[9] Puig, D., \& Garcia, M. A. (2006). Automatic texture feature selection for image pixel classification. Pattern Recognition, 39(11), 1996-2009.

[10] Kashef, S., \& Nezamabadi-pour, H. (2015). An advanced ACO algorithm for feature subset selection. Neurocomputing, 147, 271-279.

[11] Yong, Z., Dun-wei, G., \& Wan-qiu, Z. (2015). Feature selection of unreliable data using an improved multi-objective PSO algorithm. Neurocomputing.

[12] Alexandre, E., Cuadra, L., Salcedo-Sanz, S., Pastor-Sánchez, A., \& Casanova-Mateo, C. (2015). Hybridizing Extreme Learning Machines and Genetic Algorithms to select acoustic features in vehicle classification applications. Neurocomputing, 152, 58-68.

[13] Diao, R., \& Shen, Q. (2012). Feature selection with harmony search. Systems, Man, and Cybernetics, Part B: Cybernetics, IEEE Transactions on, 42(6), 1509-1523.

[14] d Gundimada, S., Asari, V. K., \& Gudur, N. (2010). Face recognition in multi-sensor images based on a novel modular feature selection technique. Information Fusion, 11, 124-132

[15] Jensen, R. (2005). Combining rough and fuzzy sets for feature selection. Ph.D. thesis, University of Edinburgh.

[16] Rashno, A., SadeghianNejad, H., \& Heshmati, A. (2013). Highly Efficient Dimension Reduction for Text-Independent Speaker Verification Based on Relieff Algorithm and Support Vector Machines". International Journal of Signal Processing, Image Processing and Pattern Recognition, 6(1).

[17] Diao, R., Chao, F., Peng, T., Snooke, N., \& Shen, Q. (2014). Feature selection inspired classifier ensemble reduction. Cybernetics, IEEE Transactions on, 44(8), 1259-1268. 
[18] Shang, C., \& Barnes, D. (2010, September). Combining support vector machines and information gain ranking for classification of mars mcmurdo panorama images. In Image Processing (ICIP), 2010 17th IEEE International Conference on (pp. 1061-1064). IEEE.

[19] Dunn, P. F. (2014). Measurement and data analysis for engineering and science. CRC press.

[20] ISO/IEC 15938-3/FDIS Information Technology-Multimedia Content Description Interface-Part 3 Visual Jul. 2001, ISO/IEC/JTC1/SC29/WG11 Doc. N4358.

[21] Gonzalez, R., Woods R.E. (2008). Digital Image Processing and Scene Analysis, third edition, Prentice Hall,.

[22] Huang, Guang-Bin. "An insight into extreme learning machines: random neurons, random features and kernels." Cognitive Computation 6.3 (2014): 376-390.

[23] Cao, Jiuwen, and Zhiping Lin. "Extreme Learning Machines on High Dimensional and Large Data Applications: A Survey." Mathematical Problems in Engineering 501 (2015): 103796.

[24] Huang, Gao, et al. "Semi-supervised and unsupervised extreme learning machines." Cybernetics, IEEE Transactions on 44.12 (2014): 2405-2417.

[25] http://marswatch.astro.cornell.edu/pancam_instrument/home_plate_south.html.

[26] Mladenić, D. (2006). Feature selection for dimensionality reduction (pp. 84-102). Springer Berlin Heidelberg.

[27] Nemati, S., \& Basiri, M. E. (2011). Text-independent speaker verification using ant colony optimization-based selected features. Expert Systems with Applications, 38(1), 620-630.

[28] Peng, Hanchuan, Fulmi Long, and Chris Ding. "Feature selection based on mutual information criteria of max-dependency, max-relevance, and min-redundancy." Pattern Analysis and Machine Intelligence, IEEE Transactions on 27.8 (2005): 12261238.

[29] 17 Liu, H., \& Motoda, H. (Eds.). (2007). Computational methods of feature selection. CRC Press.

[30] Yang, J., \& Honavar, V. (1998). Feature subset selection using a genetic algorithm. In Feature extraction, construction and selection (pp. 117-136). Springer US.

[31] Beddoe, G. R., \& Petrovic, S. (2006). Selecting and weighting features using a genetic algorithm in a case-based reasoning approach to personnel rostering. European Journal of Operational Research, 175(2), 649-671.

[32] Dorigo, M., Birattari, M., \& Stutzle, T. (2006). Ant colony optimization. Computational Intelligence Magazine, IEEE, 1(4), 28-39.

[33] Al-Ani, A. (2005, February). Ant Colony Optimization for Feature Subset Selection. In WEC (2) (pp. 35-38).

[34] Huang, C. L. (2009). ACO-based hybrid classification system with feature subset selection and model parameters optimization. Neurocomputing, 73(1), 438-448.

[35] Kanan, H. R., \& Faez, K. (2008). An improved feature selection method based on ant colony optimization (ACO) evaluated on face recognition system. Applied Mathematics and Computation, 205(2), 716-725. 